\title{
Fever, rigors and sweats in an immunocompromised male
}

\author{
G.E. Kapotsis*, Z. Daniil*, Z. Sardelis*, K. Stavrakaki*, G. Saroglou*, V. Pappa*, C. Roussos*, \\ S.A. Papiris*
}

\section{Case history}

A 63-yr-old male presented with a 20-day history of fever with chills, rigors, sweats and fatigue. He resided in the countryside and there was no history of recent travel to other countries. He had a past medical history of idiopathic pulmonary fibrosis (usual interstitial pneumonia confirmed by surgical biopsy) and the last 2 yrs he was taking daily azathioprine $100 \mathrm{mg}$ per os and methylprednisolone (actually) $12 \mathrm{mg}$ per os.

On physical examination he was pale, with a body temperature of $39.5^{\circ} \mathrm{C}$, blood pressure $130 / 90 \mathrm{mmHg}$, pulse rate 90 beats per min and respiratory rate 24 breaths per min. There were no skin rashes, petechiae or ecchymoses. The abdomen was soft, without tenderness or rigidity and the spleen was enlarged $3 \mathrm{~cm}$ below the costal margin. The auscultation revealed "velcro" rales at the lower lung fields bilaterally. There were no heart murmurs.

Laboratory investigations revealed the following values: haemoglobin $1.271 \mathrm{mM}\left(78 \mathrm{~g} \cdot \mathrm{L}^{-1}\right)$; total leukocyte count $1.54 \times 10^{9} \cdot \mathrm{L}^{-1}$, with differential count $56 \%$ polymorphonuclears,
$39 \%$ lymphocytes and $4 \%$ monocytes, and $57 \times 10^{9} \cdot \mathrm{L}^{-1}$ platelets. The prothrombin time and activated partial thromboplastin time were within normal range. The blood urea was $16 \mathrm{mM}$ the creatinine $97.2 \mu \mathrm{M}$, total bilirubin $42.75 \mu \mathrm{M}$ with a indirect fraction $27.36 \mu \mathrm{M}$, aspartate aminotransferase $1500 \mathrm{nM} \cdot \mathrm{s}^{-1}$ alanine aminotransferase $866.8 \mathrm{nM} \cdot \mathrm{s}^{-1}$. Peripheral blood smear showed no parasites. Cultures of blood and urine failed to yield any significant growth, and induced sputum was negative for Pneumocystis carinii. Mantoux and sputum test for acid-fast bacilli were negative. Serology for human immunodeficiency virus (HIV) and urine Legionnella antigen were negative, while the results of serological tests for mycoplasma, chlamydia, ricketsiae, malaria, leishmania, brucellosis and common virus were pending.

Chest roentgenogram (fig. 1a) and computed tomography scan have shown no apparent changes compared with past films (12 months previous). An upper abdomen film is visible in figure $1 b$.

A bone marrow aspirate was performed; the findings are shown in figure 2.
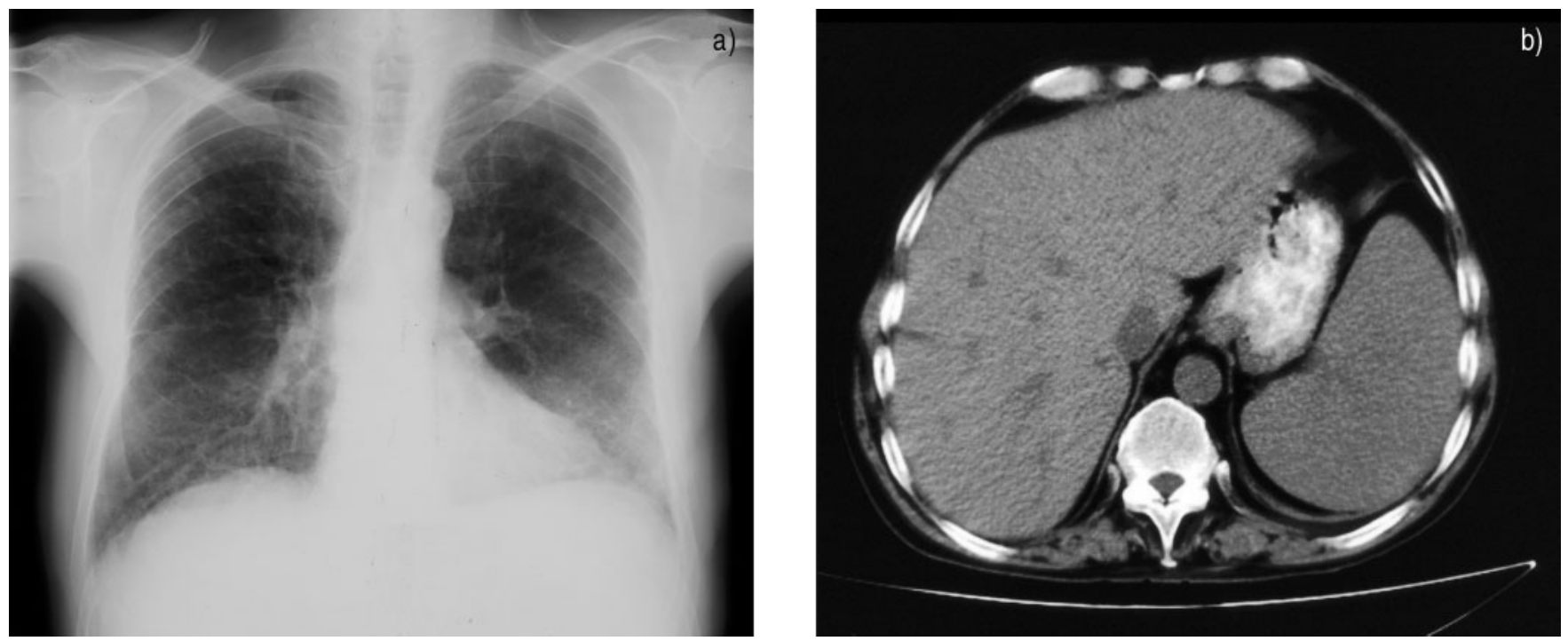

Fig. 1.-a) Chest roentgenogram and b) computed tomography, of the upper abdomen.

\footnotetext{
*Dept of Critical Care and Pulmonary Services and ${ }^{\#}$ 2nd Dept of Internal Medicine (Propedeutic), National and Capodistrian University of Athens, Evangelismos Hospital, Athens, Greece.

Correspondence: S.A. Papiris, Dept of Critical Care and Pulmonary Services, 45-47 Ipsilantou Street, Evangelismos Hospital, GR 10675, Athens, Greece. Fax: 30 2107293470. E-mail: papiris@otenet.gr
} 


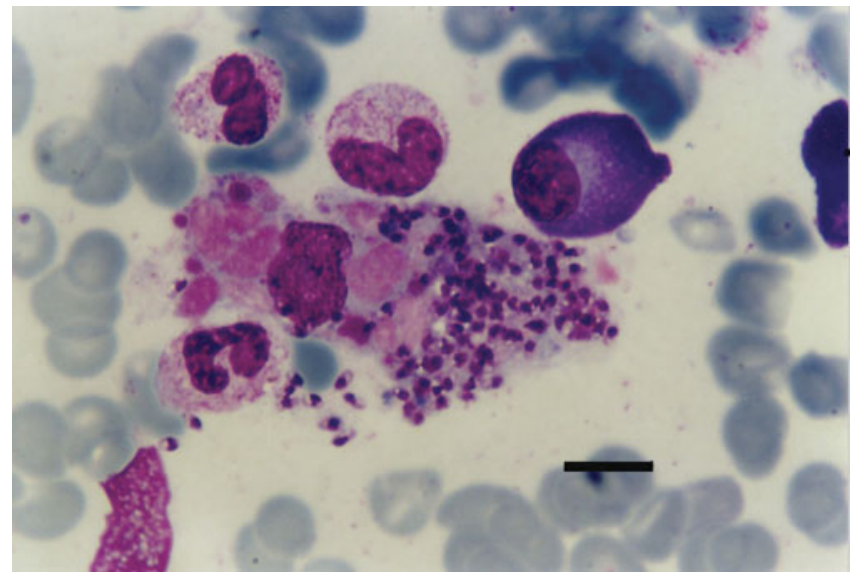

Fig. 2.-A slide examination of bone marrow aspirate (Giemsa

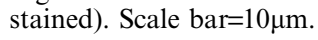

BEFORE TURNING THE PAGE, INTERPRET THE ROENTGENOGRAM, THE CT SECTION AND THE BONE MARROW ASPIRATE. 


\section{Interpretation}

The posteroanterior chest roentgenogram (fig. 1a), shows diffuse bilateral reticular opacities in the lower lung fields, compatible with the underlying disorder, idiopathic pulmonary fibrosis (IPF). The computed tomography of the upper abdomen (fig. 1b) shows an abnormally enlarged spleen.

A microscope slide examination of bone marrow aspirate (Giemsa stained) demonstrates multiple intracellular amastigotes (the tissue form of the leishmania parasite). Each amastigote has a nucleus and a kinetoplast. Some amastigotes are extracellular, probably released from mononuclear phagocytes during specimen manipulations.

Diagnosis: Visceral leishmaniasis (kala-azar) in an immunocompromised patient

\section{Clinical course}

Initially, the patient was treated empirically, with a third generation cephalosporin and a quinolone. As the diagnosis was not established yet and patient's clinical course was progressively worsening, liposomial amphotericin B was empirically added to the regimen. After the demonstration of the parasites in the infected bone marrow, medical therapy with liposomial amphotericin B was continued but his clinical course rapidly deteriorated and finally he was intubated, because of septic shock, but died soon after.

\section{Discussion}

Visceral leishmaniasis (kala-azar) is a disseminated protozoan infection, transmitted by female sand fly bites, in which macrophages of the liver, spleen and bone marrow are preferentially parasitised [1]. The genus Leishmania comprises of a growing number of species, which are zoonotic and of which $\sim 20$ cause disease in humans. Their distribution is determined by that of their vector or their reservoir host and so is dependent on precise environmental features [2]. Human infection is dependent on the ecological relationship between human activity and reservoir systems. Economic development leads to changing interactions between humans and their physical and biological environment. Increasing risk factors are making leishmaniasis a growing public health concern for many countries. According to World Health Organization statistics [3], 12 million people are affected by the disease worldwide, and 1.5-2 million new cases are estimated to occur annually. Leishmaniasis is endemic in $>80$ countries in Asia and Africa (L. donovanii), South America (L. chagasi) and southern Europe (L. infantum), spreading in several areas, as a consequence of massive urban migration and its association with HIV infection. However, most infections with $L$. donovanii or L. chagasi in the normal host are frequently asymptomatic and self-resolving.

Patients with visceral leishmaniasis are typically present with fever, cough, abdominal pain, diarrhoea, epistaxis, splenomegaly, hepatomegaly, peripheral lymphadenopathy and pancytopenia. In southern Europe, visceral leishmaniasis with the classical triad, splenomegaly, pallor and fever was reported as a childhood disease whereas today the disease may appear with atypical clinical expressions in immunocompromised patients. Idiopathic pulmonary fibrosis per se is not a predisposing condition for the development of visceral leishmaniasis. However, since many of those patients become immunocompromised because of treatment, leishmania opportunistic infection should be included in the differential diagnosis in case of fever, especially in those living in the Mediterranean area. The diagnosis is confirmed by demonstration of the parasite in the infected tissue [4]. Intracellular leishmania can be identified or cultured from aspirates of spleen, liver, bone marrow and lymph node. The diagnostic yield is highest for spleen aspirates (98\%), but there are contraindications and complications. Many centres have been evaluating the use of polymerase chain reaction, especially on peripheral blood samples [5].

Until the early 1990s and in use for 50 yrs, the mainstay of treatment worldwide was the pentavalent antimony but growing resistance over the last two decades renders this cheap and easily available drug useless. Second-line drugs (pentamidine and amphotericin B) are more toxic and difficult to administer. Newer formulations, like lipid formulations of amphotericin B and new drugs, like oral miltefosine (which recently proved even more effective than amphotericin B) [6] and paromomycin are now registered in India [4]. To date, there are no vaccines against leishmanniasis and control measures rely on chemotherapy to treat the disease and on vector control to reduce transmission [7]

\section{References}

1. Murray WH. Clinical and Experimental Advances in Treatment of Visceral Leishmaniasis. Antimicrobial Agents Chemotherapy 2001; 45: 2185-2197.

2. Ashford RW. The leismaniases as emerging and reemerging zoonoses. International Journal of Parasitology 2000; 30: 1269-1281.

3. Programme for the surveillance and control of leishmaniasis. Geneva: World Health Organization (2003). http://www.who. int/emc/diseases/leish/leishmaniasis. Date updated: March 2001; Date accessed: 2 July 2003.

4. Guerin JP, Olliaro P, Sundar S, Croff LS, Desjeux P, Bryceson DMA, et al. Visceral leishmaniasis: current status of control, diagnosis, and treatment, and a proposed research and development agenda. Lancet Infect Dis 2002; 2: 494-501.

5. Salotra P, Sreenivas G, Pogue GP. Development of a species-specific PCR assay for detection of Leishmania Donovanii in clinical samples from patients with kala-azar and post-kala-azar dermal leishmaniasis. J Clin Microbiol 2001; 39: 849-854.

6. Sundair S, Jha TK, Thakur CP, et al. Oral miltefosine for indian visceral leishmaniasis. N Engl J Med 2002; 347: 17391746.

7. Handman E. Leishmaniasis: current status of vaccine development. Clin Microbiol Rev 2001; 14: 229-243. 